\title{
New Circular model induced by Inverse Stereographic projection on Double Exponential Model - Application to Birds Migration Data
}

\author{
S.V.S.GIRIJA, A.V. DATTATREYA RAO AND PHANI YEDLAPALLI
}

\begin{abstract}
This paper introduces Stereographic Double Exponential Model based on inverse Stereographic Projection or Bilinear (Mobius) Transformation, [Minh and Farnum (2003)]. onsidering the data set of 13 homing pigeons were released singly in the Toggenburg Valley in Switzerland under sub Alpine conditions (data quoted in Batschelet (1981)), it is shown that the said model is a good fit by most of the tests at various level of significance. The derivation of the characteristic function for Stereographic Double Exponential Model and its trigonometric moments are presented. Relative performance of Stereographic Logistic [Phani (2013], Wrapped Logistic [Dattatreya Rao et al (2007)] and Stereographic Double Exponential models for the live data of 13 birds is studied. Also graphs of pdf of this new Model for various combinations of the parameters are drawn.
\end{abstract}

Mathematics Subject Classification 2000: 60E05, 62H11

Additional Key Words and Phrases: Characteristic function, Circular models, goodness - of - fit, Inverse Stereographic Projection, Trigonometric moments

\section{INTRODUCTION}

Dattatreya Rao et al (2007) constructed new circular models by applying wrapping by reducing a linear variable to its modulo $2 \pi$ and using trigonometric moments. Taking this as a cue, here an attempt is made to derive a new circular model by applying the inverse Stereographic projection on the Double Exponential /Laplace model. The density, distribution and characteristic functions of the proposed new circular model are derived.

This paper is organized as follows. Section2 describes the methodology of Inverse Stereographic Projection. In section 3 defines the proposed circular distribution, draw the graphs of density function for various parameters. In section 4 characteristic function of said model is derived, fist two trigonometric moments are derived. We conclude in section 5 with an application of said model and relative performance of Stereographic models along with one wrapped model viz., wrapped logistic model [Dattatreya Rao et al (2007)] is discussed. 


\section{METHODOLOGY OF INVERSE STEREOGRAPHIC PROJECTION}

Inverse Stereographic Projection is defined by a one to one mapping given by $T(\theta)=x=u+v \tan \left(\frac{\theta}{2}\right)$, where $x \in(-\infty, \infty), \theta \in[-\pi, \pi), u \in \square$, and $\quad v>0$ Then $T^{-1}(x)=\theta=2 \tan ^{-1}\left\{\frac{(x-u)}{v}\right\}$ by Minh and Farnum (2003) is a random point on the Unit circle. Suppose $x$ is randomly chosen on the interval $(-\infty, \infty)$. Let $F(x)$ and $f(x)$ denote the Cumulative distribution and probability density functions of the random variable $X$ respectively. Also $G(\theta)$ and $g(\theta)$ denote the Cumulative distribution and probability density functions of this random point $\theta$ on the unit circle respectively. Then $G(\theta)$ and $g(\theta)$ can be written in terms of $F(x)$ and $f(x)$ using the following Theorem 2.1 as stated below.

THEOREM 2.1: For $v>0$,

i) $G(\theta)=F\left(u+v \tan \left(\frac{\theta}{2}\right)\right)=F(x(\theta))$

ii)

$$
\begin{aligned}
g(\theta) & =v\left(\frac{1+\tan ^{2}\left(\frac{\theta}{2}\right)}{2}\right) f\left(u+v \tan \left(\frac{\theta}{2}\right)\right) \\
& =v\left(\frac{1+\left(\frac{x(\theta)-u}{v}\right)^{2}}{2}\right) f(\theta(x))
\end{aligned}
$$

\section{STEREOGRAPHIC DOUBLE EXPONENTIAL DISTRIBUTION}

A random variable $X$ on the real line is said to have Double Exponential $\backslash$ Laplace Distribution with location parameter $\gamma$ and scale parameter $\lambda>0$, if the probability density function and cumulative distribution function of $X$ for $x, \gamma \in \square$ and $\lambda>0$ are given by

$$
f(x)=\frac{1}{2 \lambda} \exp \left(\frac{-|x-\gamma|}{\lambda}\right), \lambda>0,-\infty<x<\infty
$$

and $F(x)=\frac{1}{2}\left[1+\operatorname{sign}(x)\left\{1-\exp \left(\frac{|x-\gamma|}{\lambda}\right)\right\}\right], \lambda>0,-\infty<x<\infty$

respectively. 
Then by applying Inverse Stereographic Projection defined by a one to one mapping $x=u+v \tan \left(\frac{\theta}{2}\right), u, v>0 \in \square$ and $-\pi \leq \theta<\pi$, which leads to a Circular Stereographic Double Exponential Distribution on unit circle.

\section{DEFINITION:}

A random variable $X_{S}$ on the unit circle is said to have the Stereographic Double Exponential Distribution with location parameter $\mu$ scale parameter $\sigma>0$ denoted by $\operatorname{SDEXP}(\sigma, \mu)$, if the probability density and the cumulative distribution functions are respectively given by

$g(\theta)=\frac{1}{4 \sigma} \sec ^{2}\left(\frac{\theta}{2}\right) \exp \left(-\frac{1}{\sigma}\left|\tan \left(\frac{\theta}{2}\right)-\mu\right|\right)$

where $\mu=\frac{\gamma}{v}, \quad \sigma=\frac{\lambda}{v}>0, \quad-\pi \leq \theta<\pi$,

and

$G(\theta)=(0.5)\left[1+\operatorname{sign}\left(\tan \left(\frac{\theta}{2}\right)\right)\left\{1-\exp \left(-\frac{1}{\sigma}\left|\tan \left(\frac{\theta}{2}\right)-\mu\right|\right)\right\}\right],-\pi \leq \theta<\pi$

Hence the proposed new model $\operatorname{SDEXP}(\sigma, \mu)$ is a circular model named by us as

"STEREOGRAPHIC DOUBLE EXPONENTIAL MODEL".

Graphs of the probability density function of the Stereographic Double Exponential Distribution for various values of $\sigma$ and $\mu=0$ 
Figure 1 Graph of the pdf of the Stereographic Double Exponential distribution

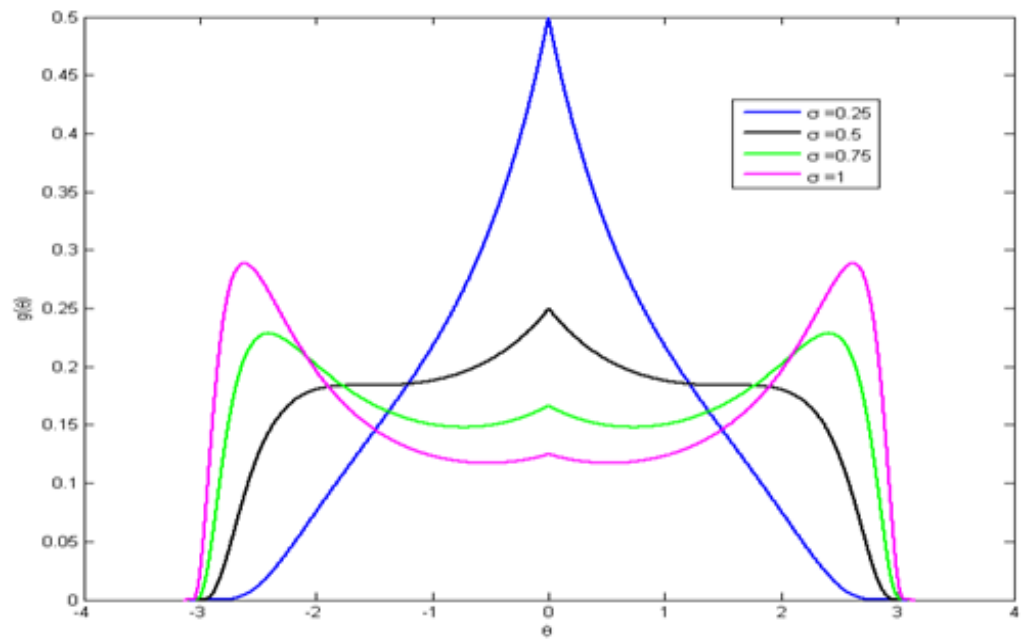

Figure 2 Graph of the pdf of the Stereographic Double Exponential distribution (Circular representation)

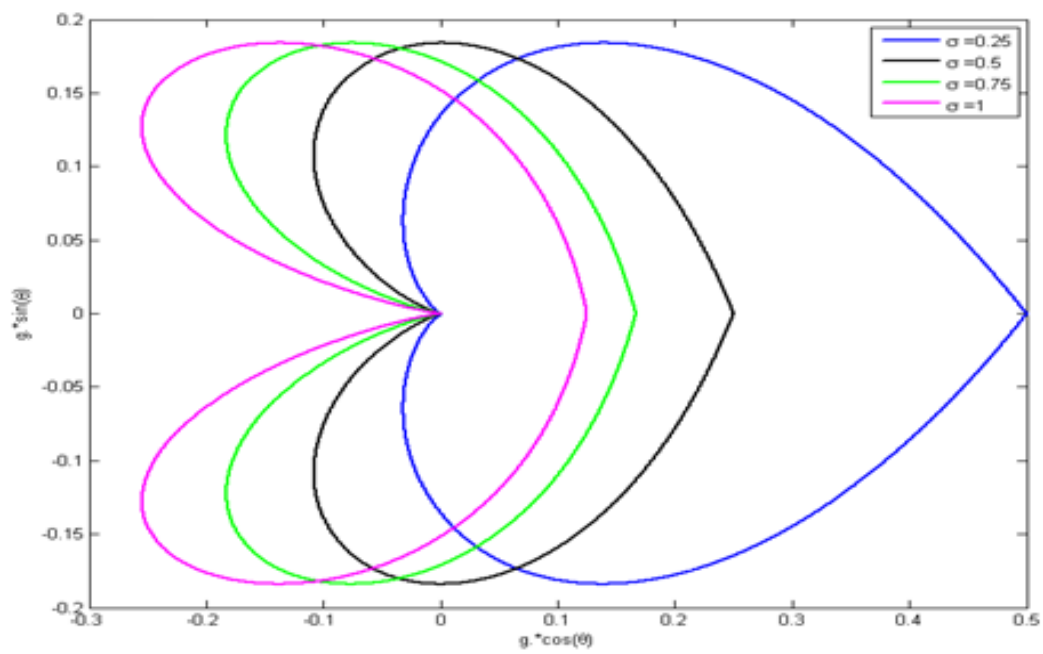

\section{THE CHARACTERISTIC FUNCTION OF STEREOGRAPHIC MODEL:}

The Characteristic function of a Circular model with probability density function $g(\theta)$ is defined as $\varphi_{p}(\theta)=\int_{0}^{2 \pi} e^{i p \theta} g(\theta) d \theta, p \in \square$. Ramabhadra Sarma et al $(2009 ; 2011)$ derived the characteristic functions of some new wrapped models based on the Proposition[c.f. p.31, Jammalamadaka and SenGupta (2001)]. This proposition cannot be applied directly in case of Stereographic Circular Model. The characteristic function of a 
Stereographic Circular model can be obtained in terms of respective linear model. Lukacs (1970) proved the following theorem related to the Characteristic function of linear model which is applied here in the case of Stereographic Circular Models.

THEOREM 4.1: Let $X$ be a random variable with distribution function $F(x)$ and suppose that $S(x)$ is a finite and single-valued function of $x$. The Characteristic function of $f_{Y}(t)$ of the random variable $Y=S(x)$ is then given by $f_{Y}(t)=E\left(e^{i t Y}\right)=E\left(e^{i t S(X)}\right)=\int_{-\infty}^{\infty} e^{i t S(X)} d F(x)$.

By applying the above theorem we derive the Characteristic function of a Stereographic Circular model.

THEOREM 4.2: If $G(\theta)$ and $g(\theta)$ are the cdf and pdf of the Stereographic Circular model and $F(x)$ and $f(x)$ are cdf and pdf of the respective linear model, then characteristic function of Stereographic Model is $\varphi_{\mathrm{X}_{S}}(p)=\varphi_{2 \tan ^{-1}\left(\frac{x}{v}\right)}(p), p \in \square$

PROOF:

$$
\begin{aligned}
\varphi_{\mathrm{X}_{S}}(p) & =\int_{-\pi}^{\pi} e^{i p \theta} d(G(\theta)), p \in \square \\
& =\int_{-\pi}^{\pi} e^{i p \theta} d\left(F\left(v \tan \frac{\theta}{2}\right)\right) \\
& =\int_{-\infty}^{\infty} e^{i p\left(2 \tan ^{-1}\left(\frac{x}{v}\right)\right)} f(x) d x, \quad \text { taking } x=v \tan \left(\frac{\theta}{2}\right) \\
& =\varphi_{2 \tan ^{-1}\left(\frac{x}{v}\right)}(p)
\end{aligned}
$$

The characteristic function of the Stereographic Double Exponential Distribution

$$
\begin{aligned}
\Phi_{X_{S}}(p) & =\int_{-\pi}^{\pi} e^{i p \theta} g(\theta) d \theta \\
& =\int_{-\pi}^{\pi} e^{i p \theta} \frac{1}{4 \sigma} \sec ^{2}\left(\frac{\theta}{2}\right) \exp \left(-\frac{1}{\sigma}\left|\tan \left(\frac{\theta}{2}\right)\right|\right) d \theta \\
& =\frac{1}{2 \sigma} \int_{0}^{\pi} \cos p \theta \sec ^{2}\left(\frac{\theta}{2}\right) \exp \left(-\frac{1}{\sigma} \mid \tan \left(\frac{\theta}{2}\right)\right) d \theta,
\end{aligned}
$$


Since $\operatorname{sinp} \theta$ is an odd function

$$
\Phi_{X_{S}}(p)=\frac{1}{2 \sigma} \int_{0}^{\pi} \cos p \theta \sec ^{2}\left(\frac{\theta}{2}\right) \exp \left(-\frac{1}{\sigma} \tan \left(\frac{\theta}{2}\right)\right) d \theta
$$

As the integral cannot be obtained analytically, MATLAB techniques are applied for the evaluation of the values of the characteristic function.

\section{Trigonometric moments of the Stereographic Double Exponential Model}

The trigonometric moments of the distribution are given by $\left\{\varphi_{\mathrm{p}}: \mathrm{p}= \pm 1, \pm 2, \pm 3, \ldots\right\}$, where $\quad \varphi_{\mathrm{p}}=\alpha_{\mathrm{p}}+i \beta_{\mathrm{p}}$, with $\quad \alpha_{\mathrm{p}}=E(\cos \mathrm{p} \theta) \quad$ and $\beta_{\mathrm{p}}=E(\sin \mathrm{p} \theta)$ being the $\mathrm{p}^{\text {th }}$ order cosine and sine moments of the random angle $\theta$, respectively. Because the Stereographic Double Exponential distribution is symmetric about $\mu=0$, it follows that the sine moments are zero. Thus $\varphi_{\mathrm{p}}=\alpha_{\mathrm{p}}$.

THEOREM 4.2. Under the pdf of Stereographic Double Exponential distribution with $\mu=0$, the first two $\alpha_{\mathrm{p}}=E(\cos \mathrm{p} \theta), \mathrm{p}=1,2$ are given as follows

$$
\begin{aligned}
& \alpha_{1}=1-\frac{1}{\sigma \sqrt{\pi}} G_{13}^{31}\left(\frac{1}{4 \sigma^{2}} \mid \begin{array}{l}
-\frac{1}{2} \\
-\frac{1}{2}, 0, \frac{1}{2}
\end{array}\right), \\
& \alpha_{2}=1+\frac{2}{\sigma \sqrt{\pi}} G_{13}^{31}\left(\frac{1}{4 \sigma^{2}} \mid \begin{array}{l|l}
-\frac{3}{2} \\
-\frac{1}{2}, 0, \frac{1}{2}
\end{array}\right)-\frac{2}{\sigma \sqrt{\pi}} G_{13}^{31}\left(\frac{1}{4 \sigma^{2}} \mid \begin{array}{c|c}
-\frac{1}{2} \\
-\frac{1}{2}, 0, \frac{1}{2}
\end{array}\right)
\end{aligned}
$$

where $\int_{0}^{\infty} x^{2 v-1}\left(u^{2}+x^{2}\right)^{Q-1} e^{-\mu x} d x=\frac{u^{2 v+2 Q-2}}{2 \sqrt{\pi} \Gamma(1-Q)} G_{13}^{31}\left(\frac{\mu^{2} u^{2}}{4} \mid \begin{array}{l}1-v \\ 1-Q-v, 0, \frac{1}{2}\end{array}\right)$ 
for $|\arg u \pi|<\frac{\pi}{2}, \operatorname{Re} \mu>0$ and $\operatorname{Re} v>0$ and $G_{13}^{31}\left(\begin{array}{l|l}\frac{\mu^{2} u^{2}}{4} & 1-v \\ 1-Q-v, 0, \frac{1}{2}\end{array}\right)$ is

called as Meijer's G-function (Gradshteyn and Ryzhik, 2007, formula no. 3.389.2).

PROOF:

$$
\begin{aligned}
& \varphi_{\mathrm{p}}=\int_{-\pi}^{\pi} e^{i p \theta} g(\theta) d \theta=\int_{-\pi}^{\pi} \cos (\mathrm{p} \theta) g(\theta) d \theta+i \int_{0}^{\pi} \sin (\mathrm{p} \theta) g(\theta) d \theta \\
& =E(\cos (\mathrm{p} \theta))=\alpha_{\mathrm{p}} \text { for } \mathrm{p}=0, \pm 1, \pm 2, \pm 3, \ldots
\end{aligned}
$$

To derive the first cosine moment $\alpha_{1}=\frac{1}{2 \sigma} \int_{0}^{\pi} \cos \theta \sec ^{2}\left(\frac{\theta}{2}\right) e^{-\frac{1}{\sigma}\left(\tan \left(\frac{\theta}{2}\right)\right)} d \theta$, we use the transformation $x=\tan \left(\frac{\theta}{2}\right), \cos \theta=1-\frac{2 x}{1+x^{2}}$ and the above integral formula

Now $\mathrm{E}(\cos (\mathrm{p} \theta))=\frac{1}{4 \sigma} \int_{-\pi}^{\pi} \cos (\mathrm{p} \theta) \sec ^{2}\left(\frac{\theta}{2}\right) e^{-\frac{1}{\sigma}\left(\tan \left(\frac{\theta}{2}\right)\right)} d \theta$

$$
\begin{aligned}
& \alpha_{1}=\frac{1}{2 \sigma} \int_{0}^{\pi} \cos \theta \sec ^{2}\left(\frac{\theta}{2}\right) e^{-\frac{1}{\sigma}\left(\tan \left(\frac{\theta}{2}\right)\right)} d \theta \\
& =\frac{1}{\sigma} \int_{0}^{\infty}\left[1-\frac{2 x^{2}}{1+x^{2}}\right] e^{-\frac{1}{\sigma} x} d x \\
& =1-\frac{2}{\sigma} \int_{0}^{\infty} x x^{2\left(\frac{3}{2}\right)-1}\left(1+x^{2}\right)^{0-1} e^{-\frac{1}{\sigma} x} d x \\
& \alpha_{1}=1-\frac{1}{\sigma \sqrt{\pi}} G_{13}^{31}\left(\frac{1}{4 \sigma^{2}} \mid-\frac{1}{2}, 0, \frac{1}{2}\right)
\end{aligned}
$$


To derive the second cosine moment $\alpha_{2}=\frac{1}{2 \sigma} \int_{0}^{\pi} \cos 2 \theta \sec ^{2}\left(\frac{\theta}{2}\right) e^{-\frac{1}{\sigma}\left(\tan \left(\frac{\theta}{2}\right)\right)} d \theta$, we use the transformation $x=\tan \left(\frac{\theta}{2}\right), \cos 2 \theta=1+\frac{8 x^{4}}{\left(1+x^{2}\right)^{2}}-\frac{8 x^{2}}{\left(1+x^{2}\right)}$ and the above integral formula.

$$
\begin{aligned}
& \alpha_{2}=\frac{1}{2 \sigma} \int_{0}^{\pi} \cos 2 \theta \sec ^{2}\left(\frac{\theta}{2}\right) e^{-\frac{1}{\sigma}\left(\tan \left(\frac{\theta}{2}\right)\right)} d \theta \\
& =\frac{1}{\sigma} \int_{0}^{\infty}\left[1+\frac{4 x^{4}}{\left(1+x^{2}\right)^{2}}-\frac{4 x^{2}}{\left(1+x^{2}\right)}\right] e^{-\frac{1}{\sigma} x} d x \\
& =1+\frac{4}{\sigma} \int_{0}^{\infty} x^{2\left(\frac{5}{2}\right)-1}\left(1+x^{2}\right)^{-1-1} e^{-\frac{1}{\sigma} x} d x-\frac{4}{\sigma} \int_{0}^{\infty} x^{2\left(\frac{3}{2}\right)-1}\left(1+x^{2}\right)^{0-1} e^{-\frac{1}{\sigma} x} d x
\end{aligned}
$$

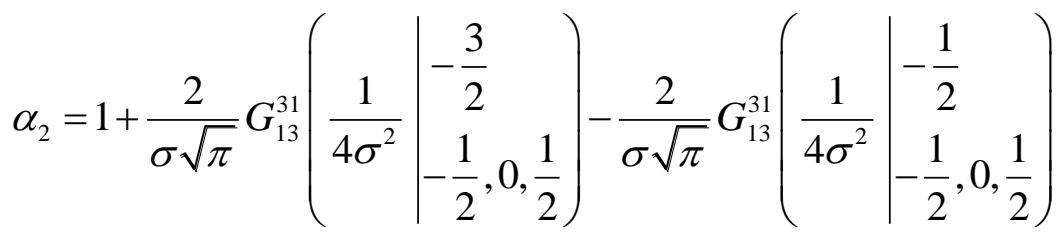

\section{APPLICATION - GOODNESS-OF -FIT FOR 13 HOMING PIGEONS DATA}

Data Set: 13 homing pigeons were released singly in the Toggenburg Valley in Switzerland under sub Alpine conditions (data quoted in Batschelet (1981)). They did not appear to have adjusted quickly to the homing direction but preferred to fly in the axis of the valley. The vanishing angles are given by the angles arranged in ascending order as follows:

$20^{0}, 135^{0}, 145^{0}, 165^{0}, 170^{0}, 200^{0}, 300^{0}, 325^{\circ}, 335^{\circ}, 350^{0}, 350^{\circ}, 350^{\circ}, 355^{\circ}$ [Jammalamadaka and SenGupta(2001), p.165]. The data plot is shown in figure 3. 
Figure 3 Graph of 13 Homing Pigeons Data Plot

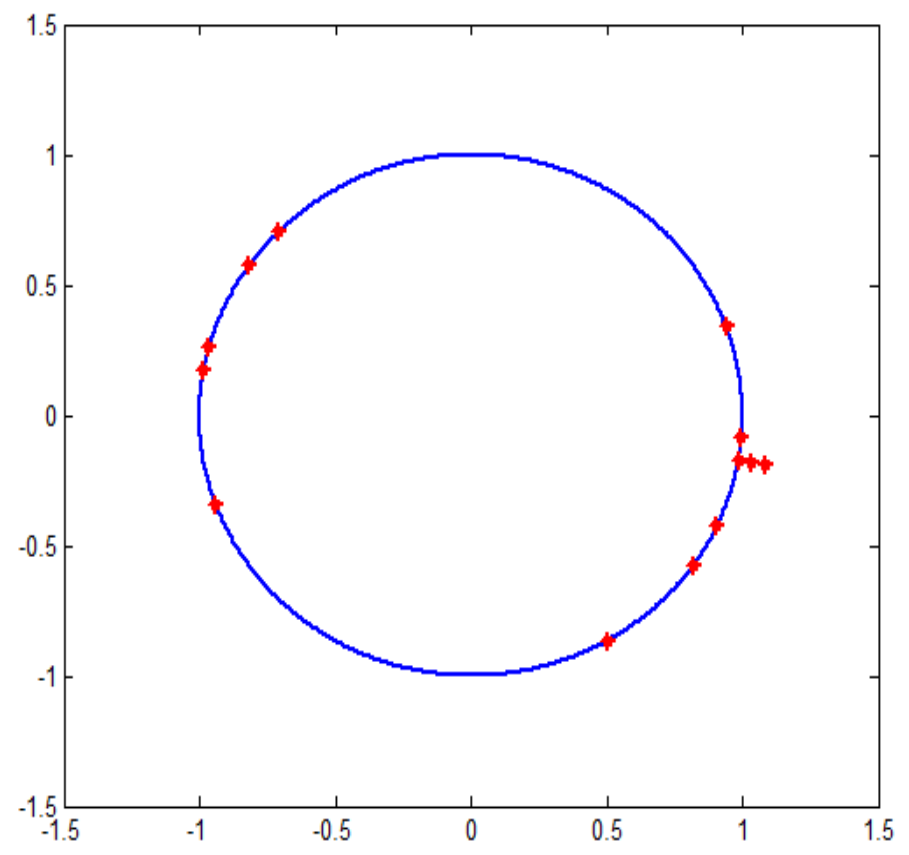

The pair of parameters can be estimated using several methods available in the literature of circular statistics. The two pairs of estimates $\hat{\mu}=-0.2735, \hat{\sigma}=0.2156$ and $\tilde{\mu}=-0.1745, \tilde{\sigma}=0.2146$ respectively.

We consider Stereographic Double Exponential, Stereographic Double Weibull [Phani (2013)] and Stereographic Logistic models [Phani (2013)] to verify goodness-of-fit for 13 homing pigeons data. We apply the following tests Viz., Rayleigh, Kuiper's, Watson's $U$ ${ }^{2}$, Hodges -Ajne, Range, Rao's Equal spacing and Ajne's tests.

The statistics of the goodness-of-fit tests mentioned above are computed for the three Stereographic circular models and are tabulated here. 
Table 1. Statistics of the Goodness-of-Fit for three Stereographic circular models

\begin{tabular}{|l|c|c|c|c|c|c|}
\hline & \multicolumn{2}{|c|}{$\begin{array}{c}\text { Stereographic } \\
\text { Logistic } \\
\text { distribution }\end{array}$} & \multicolumn{2}{c|}{$\begin{array}{c}\text { Stereographic Double } \\
\text { Weibull distribution }\end{array}$} & \multicolumn{2}{c|}{$\begin{array}{c}\text { Stereographic } \\
\text { Double Exponential } \\
\text { distribution }\end{array}$} \\
\hline $\begin{array}{l}\text { Sample Size } \\
n=13\end{array}$ & $\mu, \sigma$ & $\tilde{\mu}, \sigma$ & $\mu, \sigma, \hat{c}$ & $\mu, \sigma, \tilde{c}$ & $\mu, \sigma$ & $\tilde{\mu}, \sigma$ \\
\hline $\begin{array}{l}\text { Rayleigh } \\
\text { Test }\end{array}$ & 3.7152 & 0.7813 & 19.4611 & 17.8085 & 7.8960 & 3.4007 \\
\hline $\begin{array}{l}\text { Kuiper's } \\
\text { Test }\end{array}$ & 2.1026 & 1.7663 & 3.0696 & 2.8733 & 2.4308 & 1.9642 \\
\hline $\begin{array}{l}\text { Watson's } \\
\mathrm{U}^{2} \text { Test }\end{array}$ & 0.0079 & 0.0079 & 0.0079 & 0.0079 & 0.0079 & 0.0079 \\
\hline $\begin{array}{l}\text { Hodges-Ajne } \\
\text { Test }\end{array}$ & 9 & 9 & 10 & 9 & 10 & 9 \\
\hline Range Test & 4.6788 & 4.9706 & 2.5244 & 2.1827 & 4.4628 & 4.6158 \\
\hline $\begin{array}{l}\text { Rao's equal } \\
\text { spacing Test }\end{array}$ & 1.9279 & 1.8201 & 4.1583 & 3.3974 & 2.1750 & 1.6231 \\
\hline Ajne Test & 0.4890 & 0.2254 & 2.1076 & 1.8902 & 0.9095 & 0.5316 \\
\hline
\end{tabular}

Table 2. The cut off points for the data set of sample size $n=13$

\begin{tabular}{|l|c|c|c|}
\hline LOS & $\mathbf{1} \%$ & $\mathbf{5 \%}$ & $\mathbf{1 0 \%}$ \\
\cline { 1 - 4 } Tests & & & $0.1118-5.9786$ \\
\hline Rayleigh Test & $0.0100-10.01$ & $0.0611-6.8871$ & $0.8268-1.7709$ \\
\hline Kuiper's Test & $0.6950-2.1300$ & $0.7871-1.8691$ & $0.0229-0.1932$ \\
\hline Watson's $U^{2}$ - Test & $0.0130-0.3334$ & $0.0187-0.2300$ & $2.0000-5.0000$ \\
\hline Hodges -Ajne Test & $1.0000-6.0000$ & $1.0000-5.0000$ & $3.9930-5.3135$ \\
\hline Range Test & $3.3630-5.4925$ & $3.7381-5.3957$ & $1.5084-3.0175$ \\
\hline $\begin{array}{l}\text { Rao's Equal } \\
\text { spacing Test }\end{array}$ & $1.1610-3.4940$ & $1.3933-3.1687$ & $0.0503-0.6263$ \\
\hline Ajne Test & $0.0290-1.0436$ & $0.0396-0.7465$ & \\
\hline
\end{tabular}

On the lines of algorithm in Devaraaj (2012) the cut off points for the data set of sample size $n=13$ are computed using MATLAB techniques. 
From the above two tables it is identified that the data set follows the Stereographic Logistic and the Stereographic Double Exponential models at different levels of significance by some of the tests of Uniformity.

a) The Stereographic Logistic model is good fit as detailed below for the parameters, $\hat{\mu}=-0.2735, \hat{\sigma}=0.2156$ by

$>$ Rayleigh's, Range, Rao's equal spacing and Ajne tests at 1\%, 5\%, and $10 \%$ level of significance and Kuiper's test at 1\% level of significance only. and for $\tilde{\mu}=-0.1745$ and $\tilde{\sigma}=0.2146$ by

$>$ Rayleigh's, Kuiper's, Range, Rao's equal spacing and Ajne tests at 1\%, $5 \%$, and $10 \%$ level of significance.

b) The Stereographic Double Weibull model is not a good fit for

$$
\begin{aligned}
& \hat{\mu}=-0.2735, \hat{\sigma}=0.2156 \text { and } c=0.3136 \text { and } \tilde{\mu}=-0.1745 \\
& \tilde{\sigma}=0.2146 \text { and } \tilde{c}=0.3162
\end{aligned}
$$

c) The Stereographic Double Exponential model is good fit as detailed below for

$$
\begin{aligned}
& \hat{\mu}=-0.2735, \hat{\sigma}=0.2156 \text { by } \\
& >\quad \text { Range and Rao's equal spacing tests at } 1 \%, 5 \% \text {, and } 10 \% \text { level of } \\
& \text { significance and Rayleigh and Ajne tests at } 1 \% \text { level of significance only } \\
& \text { and for } \\
& \tilde{\mu}=-0.1745 \text { and } \tilde{\sigma}=0.2146 \text { by } \\
& >\quad \text { Rayleigh's, Range, Rao's equal spacing and Ajne tests at } 1 \%, 5 \% \text {, and } 10 \% \\
& \text { level of significance and Kuiper's test at } 1 \% \text { level of significance only. }
\end{aligned}
$$

For a given data, in a case more than one parametric circular model fits well, then choosing the appropriate model which is the best fit, can be decided by the criteria Maximum Log Likelihood (MLL), Akaike's Information Criteria (AIC) and Bayesian Information Criteria (BIC). The computations of measures of relative performances are presented. 
Table 3. Measures of Relative Performance for Goodness-of-fit at $\hat{\mu}=-0.2735$, $\hat{\sigma}=0.2156$ and $\tilde{\mu}=-0.1745, \tilde{\sigma}=0.2146$ for DataSet I

\begin{tabular}{|l|c|c|c|c|}
\hline Distribution & & MLL & AIC & BIC \\
\hline \multirow{2}{*}{$\begin{array}{l}\text { Wrapped } \\
\text { Logistic }\end{array}$} & $\hat{\mu}=-0.2735 \hat{\sigma}=0.2156$ & -82.7950 & 86.7950 & 170.7198 \\
\cline { 2 - 5 } & $\begin{array}{l}\tilde{\sigma}=-0.1745 \\
\tilde{\sigma}=0.2146\end{array}$ & -83.8172 & 87.8172 & 172.7642 \\
\hline $\begin{array}{l}\text { Stereographic } \\
\text { Logistic model }\end{array}$ & $\hat{\mu}=-0.2735 \hat{\sigma}=0.2156$ & -125.8161 & 129.8161 & 256.7620 \\
\cline { 2 - 5 } & $\begin{array}{l}\tilde{\mu}=-0.1745 \\
\tilde{\sigma}=0.2146\end{array}$ & -126.4303 & 130.4303 & 257.9904 \\
\hline $\begin{array}{l}\text { Stereographic } \\
\text { Double }\end{array}$ & $\hat{\mu}=-0.2735 \hat{\sigma}=0.2156$ & $\mathbf{- 5 5 . 7 5 3 7}$ & $\mathbf{5 9 . 7 5 3 7}$ & $\mathbf{1 1 6 . 6 3 7 2}$ \\
\cline { 2 - 5 } $\begin{array}{l}\text { Exponential } \\
\text { model }\end{array}$ & $\begin{array}{l}\tilde{\mu}=-0.1745 \\
\tilde{\sigma}=0.2146\end{array}$ & -57.4650 & 61.4650 & 120.0598 \\
\hline
\end{tabular}

Based on AIC, BIC and MLL it is unanimously identified that Stereographic Double Exponential model is the superior fit than Stereographic Logistic and Wrapped Logistic models for the estimates $\hat{\mu}=-0.2735, \hat{\sigma}=0.2156$.

\section{ACKNOWLEDGEMENT}

Authors would like to thank UGC, New Delhi, India for offering financial assistance to carry out the project under the head of Major Research Project no. F 41-785/2012 (SR) dt. 17-07-2012.

\section{REFERENCES}

1. Abramowitz, M.\& Stegun, I.A. (1965), Handbook of Mathematical Functions, Dover, New York.

2. Dattatreya Rao, A.V., Ramabhadra Sarma, I. and Girija, S.V.S. (2007), On Wrapped Version of Some Life Testing Models, Comm Statist,- Theor.Meth. , 36, issue \# 11, pp.2027-2035.

3. Dattatreya Rao, A.V., Girija, S.V.S., Phani. Y. (2011), Differential Approach to Cardioid Distribution, Computer Engineering and Intelligent Systems, Vol 2, No.8, pp. 1-6.

4. Devaraaj V. J.(2012), Some Contributions to Circular Statistics, Thesis submitted to Acharya Nagarjuna University for the award of Ph. D.

5. Girija, S.V.S., (2010), Construction of New Circular Models, VDM - VERLAG, Germany. 
6. Gradshteyn and Ryzhik (2007). Table of Integrals, series and products, 7th edition, Academic Press.

7. Jammalamadaka S. Rao and Sen Gupta, A. (2001), Topics in Circular Statistics, World Scientific Press, Singapore.

8. Lukacs, E. and Laha, R.G. (1970), Applications of Characteristic Functions, Charles Griffin and Company Limited, London, second edition.

9. Mardia, K.V. and Jupp, P.E. (2000), Directional Statistics, John Wiley, Chichester.

10. Minh, Do Le and Farnum, Nicholas R. (2003), Using Bilinear Transformations to Induce Probability Distributions, Communication in Statistics - Theory and Methods, 32, 1, pp. $1-9$.

11. Phani Y. (2013), On Stereographic Circular and Semicircular Models, Thesis submitted to Acharya Nagarjuna University for the award of Ph.D.

12. Ramabhadrasarma, I. A.V.Dattatreya Rao and S.V.S.Girija (2009), On Characteristic Functions of Wrapped Half Logistic and Binormal Distributions, International Journal of Statistics and Systems, Volume 4 Number 1, pp. 33-45.

13. Ramabhadrasarma, I. A.V.Dattatreya Rao and S.V.S.Girija (2011), On Characteristic Functions of Wrapped Lognormal and Weibull Distributions, Journal of Statistical Computation and Simulation, Vol. 81, No. 5, 579-589.

14. Toshihiro Abe, Kunio Shimizu and Arthur Pewsey, (2010), Symmetric Unimodal Models for Directional Data Motivated by Inverse Stereographic Projection, J. Japan Statist. Soc., Vol. 40 (No. 1), pp. 45-61.

Dr.S.V.S.Girija

Associate Professor of Mathematics,

Hindu College,

Guntur-A.P, INDIA.

Email: svs.girija@gmail.com

Dr. A.V. Dattatreya Rao

Professor of Statistics,

Acharya Nagarjuna University, Guntur-A.P, INDIA.

Email: avdrao@gmail.com .

Dr. Phani Yedlapalli

Associate Professor of Mathematics,

Swarnandhra College of Engineering and Technology,

Narasapur-534275, A.P, INDIA.

Email: phaniyedlapalli23@gmail.com 